Mitteilungen der Österreichischen Geographischen Gesellschaft,

153. Jg. (Jahresband), Wien 2011, S. 75-100

\title{
Ethnolinguistische Minderheiten in den Alpen Erhalt und Bedrohung autochthoner Gruppen ${ }^{1)}$
}

\author{
Ernst Steinicke, Judith W ALder, Michael Beismann und Roland LöFfLer \\ (alle Innsbruck)*
}

mit 5 Abb. und 1 Tab. im Text

\section{INHALT}

Summary .75

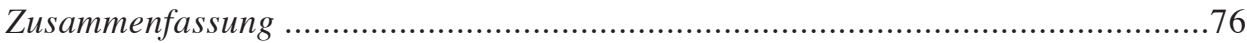

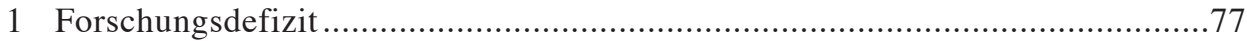

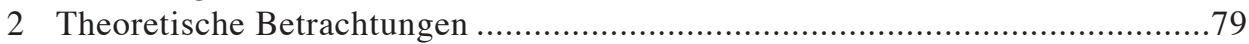

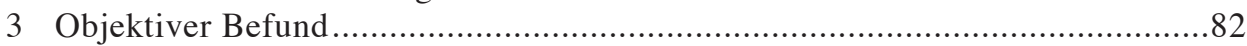

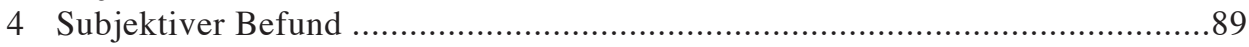

5 Bedrohung des ethnischen Mosaiks und Perspektiven ................................ 96

6 Literaturverzeichnis ............................................................................. 98

Summary

Ethno-linguistic minorities in the Alps. Endangerment to autochthonous groups and their preservation

Even though in recent decades the scientific community of geographers has increasingly engaged in discourse over ethnic issues, thus far no generally satisfying representation showing the distribution of autochthonous ethno-linguistic minorities in the Alpine region has been published. This paper attempts to fill this gap. After having set a theoretical framework, it seeks to categorize the various communities by language (as the objective characteristic of ethnicity) into national minorities, regional minorities and German-language pockets as well as to render their settlement areas

1) Die vorliegende Studie wurde vom FWF (P20954-G03) unterstützt.

* ao. Univ.-Prof. Dr. Ernst STEINICKE, Institut für Geographie, Universität Innsbruck, A-6020 Innsbruck, Innrain 52; E-Mail: ernst.steinicke@uibk.ac.at; Mag. Judith WALDER, Mag. Michael BeISMANN und Mag. Roland LÖFfLER, FWF-Arbeitsgruppe „Italienische Alpen“, Institut für Geographie, Universität Innsbruck, A-6020 Innsbruck, Innrain 52; E-Mail für alle: italian.alps@uibk.ac.at,http://www.uibk.ac.at 
cartographically. This will certainly prompt criticism, especially since also subjective factors contribute to self-identification of these minorities in addition to and in deviation from objective criteria.

It could be found that 'fuzzy ethnicities' appear in almost all minority areas. As regards the Italian part of the Alps, where most ethnic groups reside, the federal law of 1999 stipulating minority protection for areas outside the autonomous regions of Trentino-Alto Adige/Trentino-Südtirol and Valle d'Aosta/Val d'Aoste, is addressed. Since this law provides financial support for the preservation of linguistic groups, it is not really surprising that many communes with minorities whose idiom has faded away already more than a century ago and assimilated to the Italian language also declare themselves minority territories today. A second thematic map illustrates this situation, which is discussed in more detail by the example of the Dolomite Ladins.

In addition to linguistic assimilation, demographic processes can also endanger ethnic communities. In this context not so much out-migration, but rather in-migration plays the most important role nowadays. Thus, amenity migration, which endangers the conservation of ethnic diversity especially in the Italian segment of the Alps, is another issue raised. If this new phenomenon disseminates into other parts of the Alps, assimilation may also increase in these areas.

\section{Zusammenfassung}

Obwohl sich die wissenschaftliche Geographie in den letzten Jahrzehnten vermehrt mit ethnischen Fragen auseinandergesetzt hat, ist noch keine allgemein befriedigende Darstellung über die Verbreitung der autochthonen ethnolinguistischen Minderheiten im Alpenraum entstanden. Der vorliegende Beitrag versucht, diese Forschungslücke zu schließen. Nach einer theoretischen Grundorientierung war es naheliegend, die einzelnen Gemeinschaften nach dem objektiven Ethnizitätsmerkmal Sprache in nationale Minderheiten, regionale Minderheiten und deutsche Sprachinseln zu gliedern sowie ihre Siedlungsgebiete kartographisch festzuhalten. Eine solche Darstellung findet allerdings auch Kritik, zumal bei den einzelnen Minderheiten subjektive ethnische Identifikationen vorhanden sind, die von den objektiven Kriterien abweichen. Die Studie geht daher auch dieser Fragestellung nach.

Dabei ließ sich erkennen, dass solche „diffuse Ethnizitäten“ in fast allen Minderheitengebieten auftauchen. Im italienischen Alpenanteil, wo die meisten ethnischen Gruppen siedeln, wird darüber hinaus auf das Gesetz von 1999 eingegangen, das den Minderheitenschutz für die italienischen Gebiete außerhalb der autonomen Regionen Trentino-Südtirol/Trentino-Alto Adige und Aostatal [Valle d'Aosta/Val d'Aoste] regelt. Da es für den Erhalt der einzelnen Gruppen finanzielle Zuschüsse vorsieht, verwundert es nicht, dass sich zahlreiche Gemeinden, in denen die ethnische Sprache schon vor über einem Jahrhundert an das Italienische assimiliert wurde, nun ebenso zu Minderheitenterritorien deklarieren. Eine zweite thematische Karte veranschaulicht diese Situation, welche am Beispiel der Dolomitenladiner näher diskutiert wird. 
Neben der Assimilation bilden auch die demographischen Verhältnisse eine Bedrohung für den Erhalt der einzelnen Gemeinschaften. Das Letztere ist heute kaum noch mit Abwanderung in Verbindung zu bringen, sondern mit der neuen Zuwanderung in die Alpen. In diesem Zusammenhang wird die Amenity Migration, die wiederum im italienischen Alpenanteil die Bewahrung der Ethnodiversität gefährdet, thematisiert. Breitet sich dieses neue Phänomen ebenso in den übrigen Alpenteilen aus, so ist auch dort mit einer verstärkten Assimilierung zu rechnen.

\section{Forschungsdefizit}

In den meisten Teilen der Erde wird die Sprache als objektives Merkmal herangezogen, um autochthone Ethnizitäten, also bodenständige Völker und Volksgruppen, voneinander zu unterscheiden. In diesem Sinne gibt eine Sprachenverteilungskarte die ethnische Struktur in einem gewissen Gebiet wieder.

Es ist hinlänglich bekannt, dass abgelegene, kleingekammerte Gebirgsräume günstige Bedingungen für den Erhalt von ethnischen Gruppen bieten. Dementsprechend ist nirgendwo im westlichen Europa das ethnolinguistische Bild so bunt wie im Alpenbogen: Hier sind nicht weniger als elf verschiedene Sprachen beheimatet. Sieht man von subjektiven Ethnizitätsfaktoren (z.B. Bekenntnis, ethnische Identität) ab, so bilden ihre Sprecher elf autochthone ethnische Gruppen: Slowenen, Italiener, Franzosen, Deutsche, Kroaten, Ungarn, Ladiner, Rätoromanen, Friulaner, Okzitanen (Provenzalen) und Frankoprovenzalen. Damit sind die meisten Alpenländer mehr oder weniger mit Volksgruppen- oder Minoritätenfragen konfrontiert, ob sie es wahrhaben wollen oder nicht. $\left.{ }^{2}\right)$ Nicht immer ist eine Minderheitenregelung erzielt worden, die hinreichenden Schutz vor Assimilierung gewährt. Dies stellt einerseits ein permanentes Konfliktpotenzial dar, andererseits drohen dadurch kleine Volksgruppen und damit Kulturen oder ethnische Identitäten zu verschwinden (Moseley 2010).

Aufgrund ihrer kulturellen sowie gesellschaftlich-politischen Relevanz besteht heute in Europa einhelliges Bekenntnis zur Bewahrung ethnolinguistischer Minderheiten. Selbst Frankreich, dessen Verfassung die Existenz solcher Sondergruppen innerhalb seines Territoriums nicht erlaubt, hat in den letzten Jahrzehnten etliche juristischkonstitutionelle Handlungsspielräume entdeckt, um die sogenannten Regionalsprachen innerhalb des Hexagons zu schützen (SANGUIN 1993). Beispielsweise ist Korsika [Corse] de facto bereits teilautonom, und in der Bretagne gelang es, durch kulturelle Zugeständnisse militante Aktivitäten zu stoppen.

Konnte KraAs im Jahr 1992 noch ein Defizit der geographischen Forschung zur Minoritätenproblematik ausmachen (KRAAs 1992b), so hat sich die Situation bis heute

\footnotetext{
2) Die meisten Minderheitengebiete besitzen Toponyme in mehreren Sprachvarianten: Neben der staatlichoffiziellen Bezeichnung besteht in der Regel ein Name in der jeweiligen Minderheitensprache, häufig noch einer im ortsüblichen oder regionalen Dialekt. Um den Text nicht zu strapazieren, werden im Folgenden einerseits die amtlichen Ortsnamen, andererseits - falls gebräuchlich - die deutschen Exonyme verwendet.
} 
geändert, und die wissenschaftliche geographische Community liefert wesentliche neue Erkenntnisse über territoriale, demographische oder sozioökonomische Entwicklungen bei den verschiedensten Minderheiten. Angesichts der zahlreichen ethnischen Konflikte, der Beeinflussung bilateraler Beziehungen durch nationale Minderheiten sowie - innerhalb eines Staates - die Auswirkung von ungelösten Ethnizitätsfragen auf das politische und soziale Klima ist diese Zuwendung nicht überraschend: Minderheitenprobleme betreffen häufig Periphergebiete, die auch im Zentrum der EU-Regionalförderung stehen. Darüber hinaus finden sich in den Alpen ethnische Minderheiten nicht selten in den innersten Talbereichen, was Minderheiten- und Tourismusforschung zusammenrücken lässt und Minderheitenfragen eng mit Raumplanungsproblemen verknüpft. Zudem hat sich die wissenschaftliche Geographie in den letzten Jahren auch mit Fragen der ethnischen Identität und der ethnisch-emotionalen Bindung an den Raum auseinandergesetzt.

Umso mehr ist es verwunderlich, dass gerade über die autochthonen Sprachminderheiten in den Alpen noch ein erhebliches Forschungsdefizit besteht. Obwohl diverse Fachbereiche - in erster Linie die Sprachwissenschaften - eine Fülle an Beiträgen zu einzelnen Minderheitengruppen liefern, fehlt bis heute noch eine Gesamtschau über ihre tatsächliche Verbreitung. Ethno-geographische Studien in Ostmitteleuropa und Osteuropa haben gezeigt, dass eine räumliche Bestandsaufnahme nicht akademischen Interessen allein dient, sondern unerlässlich für Schutzmaßnahmen zur Bewahrung der Minoritäten ist (z.B. KraAs \& Stadelbauer 2002, Jordan 2002 oder Heller 2007). Primäres Ziel der vorliegenden Arbeit ist es daher, diese Forschungslücke zu schließen und eine kartographische Darstellung vorzulegen. Dabei sind neben dem objektiven sprachwissenschaftlichen Befund ebenso subjektive Ethnizitätsfaktoren zu berücksichtigen. Mit dem zuletzt genannten Aspekt hängt auch der gegenwärtig feststellbare Rückgang der ethnolinguistischen Diversität im Alpenraum zusammen. Als zweite Aufgabe dieses Beitrags sollten somit die raumbedeutsamen Kräfte diskutiert werden, welche den Erhalt oder das Verschwinden von Minderheiten steuern.

Annäherungen an eine entsprechende kartographische Umsetzung finden sich unter anderem im Atlas narodov mira (AKADEMIJA NAUK SSSR 1964, S. 36f.), bei STRAKA (1970), Salvi (1975), Allardt (1979), Rougier et al. (1984) oder Pan \& Pfeil (2000) sowie in den sprachwissenschaftlichen Darstellungen von Pellegrini (1977), Telmon (1992) oder Toso (2008). Aufgrund der gewählten Maßstabsebene - Europa, Alpenraum bzw. Italien und angrenzende Gebiete - beschränken sie sich jedoch zumeist auf eine schematische Darstellung. Kleine Sprachgruppen und -inseln werden zum Teil nur symbolisch gekennzeichnet und sind dadurch - schon aufgrund der Generalisierung räumlich schwer zuordenbar. Auch sich überlappende linguistische Strukturen, wie in Südkärnten, in den deutschen Sprachinseln, in der provenzalisch-frankoprovenzalischen, ladinisch-friulanisch-venezianischen bzw. provenzalisch-piemontesischen Sprachkontaktzone, wo zudem subjektive ethnische Identifikationen im Vordergrund stehen, erschweren eine exakte Abgrenzung der Volksgruppen voneinander. Das Raumbild wird schließlich durch amtliche Verordnungen, welche objektive wie auch individuellsubjektive Ethnizitätskriterien weitgehend unbeachtet lassen, weiter verkompliziert. 
Für den italienischen Alpenanteil, wo die ethnolinguistische Struktur am vielfältigsten ist, wurde kürzlich eine Minoritätenstudie vorgelegt, die auch subjektive Komponenten sowie den aktuellen demographischen Wandel berücksichtigt (W ALDER et al. 2010). Diese „Inseldarstellung“ soll hier ergänzt werden. Damit versteht sich die vorliegende Arbeit als Beitrag zu einer problemorientierten Gesamtschau über die autochthonen Minderheiten im Alpenraum. Um die inner- und interethnische Komplexität erfassen zu können, werden neben einer theoretischen Betrachtung und Bestandsaufnahme auch Problembiographien ausgewählter sprachlicher Minderheiten diskutiert. Den Abschluss bildet die Darstellung möglicher Zukunftsbilder über die Ethnodiversität in den Alpen.

Diese Studie basiert größtenteils auf Auswertung des bisherigen Forschungsstandes, wobei dafür die Vorarbeiten von Innsbrucker Geographen eine wesentliche Grundlage bilden. Dies gilt für den österreichischen, slowenischen, vor allem aber für den italienischen Alpenanteil. So fließen hier Ergebnisse sowohl von einem kürzlich abgeschlossenen als auch von einem laufenden FWF-Projekt ein. ${ }^{3)}$ Die Eigenerhebungen erfolgten im Zuge von Forschungsaufenthalten bei den ethnischen Minoritäten (2010/11). In ausgewählten Minderheitengebieten wurden Experten über die ethnische Abgrenzung zum Nachbarraum befragt. Dabei handelte es sich vornehmlich um Verantwortliche in den Gemeinden sowie um Repräsentanten verschiedener Kulturvereine. Demographische und sozioökonomische Informationen ließen sich schließlich über die jeweilige amtliche Statistik abrufen.

Das Untersuchungsgebiet - die Alpen - wurde nicht nach streng topographischgeomorphologischen Gesichtspunkten, sondern nach den Kriterien der EURAC (2004) abgegrenzt. Diese pragmatische Vorgangsweise führte allerdings dazu, dass Teile der Burgenland-Kroaten und -Ungarn, die man gemeinhin nicht zu den Minderheiten in den Alpen rechnet, als solche auszuweisen sind.

\section{Theoretische Betrachtungen}

Zunächst erscheint es notwendig, den bereits mehrfach verwendeten Begriff ,ethnische Minderheit“" zu erörtern. Abgesehen von der Schwierigkeit, eine „Minderheit“ befriedigend zu definieren (vgl. z.B. KraAs-Schneider 1989, HecKmann 1992, HutchinSON \& SMith 1996 oder Plasseraud 1998), ist es unmöglich, den politischen Ausdruck „ethnische Minderheiten“ allgemein gültig zu definieren (vgl. dazu die Literaturübersicht bei Steinicke 2005). Die juristische Sprachpraxis unterscheidet sich oftmals von Staat zu Staat. Dies betrifft vor allem die autochthone Situation. So bilden in Mitteleuropa Gastarbeiter und Immigranten aus der Türkei oder aus Südosteuropa zwar ethnische Gruppen, sie werden aber nicht als „ethnische Minderheiten“ bezeichnet, weil ihre

3) FWF-Projekt (P16664-G03, 2004-2007): The Threat to Ethno-Linguistic Diversity in the Valcanale (Italy). FWF-Projekt (P20954-G03, 2010-2012): The Impact of Current Demographic Transformation on Ethno-Linguistic Minorities in the Italian Alps. 
Ansässigkeit noch keine drei Generationen andauert (VEITER 1984). In den Niederlanden, den USA, im Vereinigten Königreich und in Kanada besteht demgegenüber eine völlig andere Sichtweise, in der Immigranten durchaus zur Kategorie ,ethnische Minderheiten“ zählen können. Einen Sonderfall stellt Frankreich dar, das offiziell auf seinem Staatsgebiet keine ethnischen Minderheiten kennt - sieht man einmal von der bereits erwähnten de facto-Situation bei den Korsen ab.

Den folgenden Ausführungen liegt das mitteleuropäische Begriffsverständnis zugrunde. Dementsprechend spielt neben dem Bekenntnis die Bodenständigkeit der sprachlichen Sondergruppen (,autochthone ethnolinguistische Gruppen“) die zentrale Rolle bei der Definition ethnischer Minderheiten. Dies gilt auch für Frankreich, wo im südlichen Alpenanteil die Provenzalen (Okzitanen) zu finden sind. Aufgrund der besonderen Einstellung zur „Égalité“, wonach keinem Individuum oder Kollektiv mehr Rechte als dem anderen zustehen dürfen, verwehrt die französische Verfassung den „Sprechern von Regionalsprachen“ die Anerkennung als ethnische Minderheit (SANGUin 1993). ${ }^{4}$

Modelle der Entwicklung von Minderheitenterritorien wurden bereits mehrfach vorgestellt (zuletzt bei STEINICKE 2001a; vgl. dazu auch KrAAs 1992a, S. 314f.). Abgesehen von wenigen Ausnahmen (z.B. Südtirol [Südtirol/Alto Adige]) ist für die meisten Minderheiten eine Territorialregression mit einherschreitender Abnahme der Zahl der Minderheitsangehörigen bezeichnend. Diese Einengung von Siedlungsgebieten wird einerseits durch Assimilation an die Majorität, also vor allem durch den Sprachwechsel, verursacht, andererseits können dafür ebenso demographische Veränderungen der betreffenden Gruppe - Migrationen oder generatives Verhalten - verantwortlich sein.

Die räumliche Peripherisierung bzw. die Verlagerung des Minderheitenterritoriums in Richtung Staatsgrenze lässt sich im Alpenraum besonders gut feststellen. Mit der Industrialisierung erfolgten aber auch in solchen abgeschiedenen Lagen letztendlich Abwanderungen in die Zentralräume, welche die Minderheiten quantitativ und qualitativ schwächten.

Der spätmoderne Strukturwandel hat nun ebenfalls in den Siedlungsgebieten der ethnischen Minderheiten eingesetzt, und Tourismus, Handel und andere Dienstleistungsbranchen sind dort oftmals zu den bedeutendsten Wirtschaftssektoren geworden. Angesichts einer fortschreitenden europäischen Integration können gerade die Regionen an der Peripherie der einzelnen Staaten mit erheblichen wirtschaftlichen Gunstfaktoren aufwarten. Die Randlage bietet dem Tourismus und jeglicher Art der grenzüberschreitenden Kooperation günstige Standorte. Nachteile, die in der Phase der Industrialisierung Gültigkeit besaßen, schwächen sich damit allmählich ab. Randgebiete mit Minderheiten können folglich nicht nur in kultureller, sondern auch in wirtschaftlicher Hinsicht Brückenfunktionen übernehmen (STEINICKE \& Zupančıč 1995).

\footnotetext{
4) Mit dieser Rechtsmeinung nimmt Frankreich innerhalb Europas eine isolierte Stellung ein. Die meisten modernen Staaten folgen dem juristischen Prinzip der ,positiven Diskriminierung“, bei der die gleiche Behandlung von Ungleichem als ungerecht erachtet wird (VEITER 1984, OpITz 2007). Trotzdem lässt sich gerade in Frankreich feststellen, dass die Kommunen den „Sprechern von Regionalsprachen“ durchaus weitreichende kulturelle Ausnahmeregelungen (Medien, Ortstafeln) gewähren. In der U-Bahn von Toulouse erfolgt beispielsweise die Ankündigung aller Stationen zweisprachig (Französisch/Okzitanisch).
} 
Strategien, welche die verschiedenen Alpenstaaten seit dem Zweiten Weltkrieg anwenden, um ihre ethnischen Minderheiten zu erhalten, sind nicht nur von Land zu Land unterschiedlich, sondern auch innerhalb von Staaten und Regionen (PAN \& PFEIL 2000). Im Alpenraum reichen sie von kulturellen Ausnahmeregelungen über demokratische Mitbestimmung bis hin zu sektoralen Autonomien oder auch zur Territorialautonomie für Südtirol und dem Aostatal. Im Burgenland, in Kärnten und Südtirol bestehen zudem internationale Vereinbarungen, welche den Minderheitenschutz verlangen (vgl. STEININGER 2003, ÖSTERREICHISCHES VOLKSGRUPPENZENTRUM 2007).

Italien hat 1999 eine bemerkenswerte Regelung für alle seine autochthonen linguistischen Minderheiten („Minoranze linguistiche storiche“) erlassen. Damit kommt der Apenninenstaat, wenn auch mit über 40-jähriger Verspätung, den in den Artikeln 3 und 6 der italienischen Verfassung ausgedrückten Bestimmungen nach. Das Ausführungsgesetz von 1999 (Nr. 482) fördert kommunale Maßnahmen, die dem Erhalt der ethnischen Vielfalt dienen (Gazzetta Ufficiale n. 297, 20.12.1999). ${ }^{5)}$ Demzufolge anerkennt Italien zwölf historische Sprachminderheiten, wobei sowohl Sprache, Staatsbürgerschaft als auch Bodenständigkeit als ausschlaggebende Kriterien für die Zugehörigkeit herangezogen werden. Für viele kleine Gruppen ist diese Regelung aber wahrscheinlich zu spät eingeführt worden. Auch in Kärnten, wo der Staatsvertrag von 1955 einen umfassenden Schutz für die slowenische Volksgruppe vorsieht, hat man die Einengung des slowenischen Siedlungsgebietes nicht aufhalten können.

Die Schweiz kennt laut Bundesverfassung vier Sprachgruppen. Dabei bilden aber die Rätoromanen in Graubünden de facto eine echte ethnolinguistische Minorität. Trotz minderheitenfreundlicher Kantonsverfassung schreitet die deutsche Sprache auf Kosten des Rätoromanischen voran (KraAs 1992a, S. 52f.; Lüd \& WERLEN 2005, S. 98f.). Die kleine deutsche Sprachinsel im Tessin [Ticino], Bosco Gurin, kann sich dagegen nicht auf einen kantonalen Schutz berufen. Slowenien hat wiederum für seine italienische und ungarische Volksgruppe spezielle Maßnahmen gesetzt, um sie vor Assimilierung zu bewahren. Die rund 1.800 Deutschsprachigen, die vor allem in der Untersteiermark [Štajersko] leben, werden jedoch von offizieller Seite (noch) nicht als ethnolinguistische Minderheit anerkannt (ANDERWALD et al. 1997, S. 139-192; KARNER 2001, Europarat 2010).

Eine Sprache stirbt dann, wenn sie die Kinder nicht mehr sprechen. Zentrale Bedeutung für ihre Erhaltung, oder umgekehrt, für ihre Zurückdrängung, haben somit Kindergarten und Schule sowie die weiteren kulturpolitischen Instrumente der staatstragenden Mehrheit, wie etwa Medien oder Verwaltungssprache. Daneben erfolgte der Einfluss der Majorität auf die Minorität vor allem über die kulturellen Traditionen des Mehrheitsvolks sowie über die Wirtschaft. Weitere Faktoren, die eine Bereitschaft zur Assimilation erhöhen oder verringern können, sind zum Beispiel Verkehrserschließung und Kommunikation, die Lage zum kulturellen Kernland, Sprachverwandtschaft, dialektale Aufsplitterung, Kodifizierungsstand der Schriftsprache sowie moderne Trends in Sport, Mode und Musik.

\footnotetext{
5) Zudem bestehen spezielle Maßnahmen seitens der Regionen, wie z.B. für die gesamte slowenische Sprachgruppe (Regionalgesetz Nr. 26/2007) oder auch für die Friulaner (Regionalgesetz Nr. 29/2007).
} 
Wie an anderer Stelle ausgeführt (STEINICKe 1991, S. 13-17), ist aber für die endgültige Assimilation der jüngeren Generation letztlich die Sprachpraxis der Erziehenden, also der Eltern, entscheidend. Da in sprachlich gemischten Ehen vorwiegend das Idiom der Majorität gepflegt und schließlich auf die Kinder übertragen wird, gehören die Jugendlichen bereits mehrfach der Sprachgruppe des staatstragenden Volkes an. So sind auch in den Alpen Sprachgrenzen nicht mehr rein topographisch aufzufassen, sie ziehen sich heute mitten durch Familien.

Für die Darstellung der vielschichtigen Minderheitenprobleme eignet sich das relativierte konstruktivistische Ethnizitätskonzept, wie es auch Heller (2007) und mit ihm SALlanz (2007) für ihre Forschungen in Südosteuropa verwendet haben. Ethnizität wird demnach einerseits als Ergebnis eines gesellschaftlichen Konstruktionsprozesses erklärt, andererseits kann man davon ausgehen, dass objektive Unterscheidungsmerkmale wie Abstammung, Verwandtschaft, Sprache oder Religion, vorhanden sein müssen, Ethnizität daher ebenso primordial angelegt ist. Ethnizität lässt sich demzufolge als Konstrukt und Realität zugleich betrachten (SAllanz 2007, S. 32).

\section{Objektiver Befund}

Im Folgenden sollen die ethnischen Minderheiten nach der Sprache, dem bedeutendsten der objektiven Ethnizitätskriterien, gegliedert werden. Für die folgenden Unterabschnitte empfiehlt es sich, die Grundsystematik von KLoss (1969, S. 62) zu übernehmen. Er unterteilt ethnische Minderheiten einerseits in Außengruppen oder nationale Minderheiten, andererseits in Eigengruppen. Im Alpenraum ist es darüber hinaus zweckmäßig, noch separat die Kategorie der deutschen Sprachinseln auszuweisen.

Abbildung 1 veranschaulicht alle ethnolinguistischen Minoritäten der Alpen. Mit farbigen Flächen sind dabei jene Siedlungsgebiete dargestellt, in denen heute noch die traditionelle Sprache im Alltag Verwendung findet. Die Punktraster weisen wiederum auf ehemalige Siedlungsgebiete von Minoritäten hin, wo die Minderheitensprache seit rund 50 Jahren keine Rolle im Alltagsleben mehr spielt. Diese Signatur wurde auch für die Minderheitenterritorien im alpinen Frankreich gewählt. Zwar ist das Provenzalische in Peripherlagen bei der ältesten Generation noch fallweise in Gebrauch, doch besitzt das Idiom im Alltagsleben keinen Stellenwert mehr; es lebt zumeist nur in regionaler Färbung des Französischen fort. Letzteres gilt auch für den nördlich daran angrenzenden frankoprovenzalischen Sprachraum Frankreichs, in dem sich allerdings nie eine eigene ethnische Identität entwickelt hat. In Anlehnung an BERSCHIN et al. (1978, S. 274-277) ist er als linguistische Besonderheit, keinesfalls jedoch als Minderheitengebiet zu betrachten.

Die vorgelegte Karte lokalisiert nicht jene Gebiete, in denen Minderheiten lediglich als historische Reminiszenz vorkommen, wie zum Beispiel schon längst verschwundene deutschsprachige, slowenische (alpenslawische) oder rätoromanisch-ladinische Territorien in den Südalpen. Dort mag die alte Kultur durchaus noch im Brauchtum, in der traditionellen Architektur oder in Ernährungsgewohnheiten fortleben. Autochtho- 
ne ethnosoziale Gruppen, wie die Fahrenden - so die Jenischen (Karrner) im Tiroler Oberinntal, im Vinschgau, in Graubünden, in Südwestdeutschland und Oberbayern (vgl. SPISs 2001) -, ferner ethnoreligiöse Gemeinschaften (z.B. Juden) sowie Roma und Sinti werden ebenso nicht dargestellt.

Tabelle 1 zeigt eine ungefähre Zahl der Minderheitenangehörigen in den verschiedenen Alpenstaaten. Auf Schwierigkeiten und Unzulänglichkeiten von Spracherhebungen und -schätzungen wird hier jedoch nicht eingegangen (vgl. dazu STEINICKE \& ZuPANČıČ 1994, 1995). Eine amtliche Zählung der Minderheiten nach dem Sprachgebrauch erfolgte in Österreich, Slowenien und in der Schweiz im Rahmen des jeweiligen letzten Zensus in den Jahren 2000/01. In Italien finden seit 50 Jahren keine amtlichen Sprachzählungen mehr statt, sieht man von Südtirol ab, in dem ebenfalls alle zehn Jahre die Zahl der Deutsch- und Ladinischsprachigen erhoben wird (zuletzt 2011). Frankreich kennt in seinem Territorium (France Métropolitaine) keine Feststellung der Sprachen.

\begin{tabular}{|c|c|c|c|}
\hline Sprachgruppe & Gebiet & Sprecherzahl (ca.) & Quelle \\
\hline $\begin{array}{l}\text { Frankoprovenzalisch/ } \\
\text { Französisch }\end{array}$ & Aostatal & 70.000 & SALVI (1975), ORIOLES (2003), eigene Modif. \\
\hline $\begin{array}{l}\text { Frankoprovenzalisch/ } \\
\text { Französisch }\end{array}$ & Piemont & 20.000 & $\begin{array}{l}\text { SALVI (1975), KATTENBUSCH (1997), ORIOLES } \\
\text { (2003), ALLASINO et al. (2007), eigene Modif. }\end{array}$ \\
\hline $\begin{array}{l}\text { Okzitanisch/ } \\
\text { Provenzalisch }\end{array}$ & Piemont & 40.000 & $\begin{array}{l}\text { KATTENBUSCH (1997), ORIOLES (2003), eigene } \\
\text { Modif. }\end{array}$ \\
\hline Walserdeutsch & Aostatal & 600 & eigene Schätzung \\
\hline Walserdeutsch & Piemont & 400 & ZÜRRER (1999), eigene Modif. \\
\hline Walserdeutsch & Tessin (Bosco Gurin) & 23 & STATISTIK SCHWEIZ, Volkszählung 2000 \\
\hline Rätoromanisch & Graubünden & 27.038 & STATISTIK SCHWEIZ, Volkszählung 2000 \\
\hline Deutsch & Südtirol & 300.000 & ASTAT (2002), eigene Modif. \\
\hline $\begin{array}{l}\text { Deutsche Sprachinseln } \\
\text { (Ostalpen) und Kanaltal }\end{array}$ & Friaul und Venetien & 3.400 & STEINICKE et al. (2006), eigene Schätzung \\
\hline Friulanisch & Friaul (Alpenanteil) & 60.000 & STEINICKE (1991), eigene Modif. \\
\hline Ladinisch & Sellagebiet & 33.000 & $\begin{array}{l}\text { KATTENBUSCH (1997), ASTAT (2002), eigene } \\
\text { Modif. }\end{array}$ \\
\hline Ladinisch & Comelico & 6.000 & eigene Schätzung \\
\hline Slowenisch & Kanaltal, Resia, Slavia & 10.000 & STEINICKE (1991), eigene Modif. \\
\hline Slowenisch & Kärnten & 13.109 & STATISTIK AUSTRIA, Volkszählung 2001 \\
\hline Slowenisch & $\begin{array}{l}\text { Steiermark (Deutsch- } \\
\text { landsberg \& Leibnitz) }\end{array}$ & 279 & STATISTIK AUSTRIA, Volkszählung 2001 \\
\hline Burgenlandkroatisch & Burgenland (Alpenanteil) & 521 & STATISTIK AUSTRIA, Volkszählung 2001 \\
\hline Ungarisch & Burgenland (Alpenanteil) & 427 & STATISTIK AUSTRIA, Volkszählung 2001 \\
\hline
\end{tabular}

* In der Steiermark sind die Daten nur für Politische Bezirke verfügbar. In den Gemeinden mit Alpenanteil (Soboth, Aibl und Schlossberg) ist die Zahl der Slowenischsprecher somit wesentlich geringer als in der Tabelle angegeben.

Tab. 1: Zahlenmäßige Größe der ethnolinguistischen Minderheiten im Alpenraum 


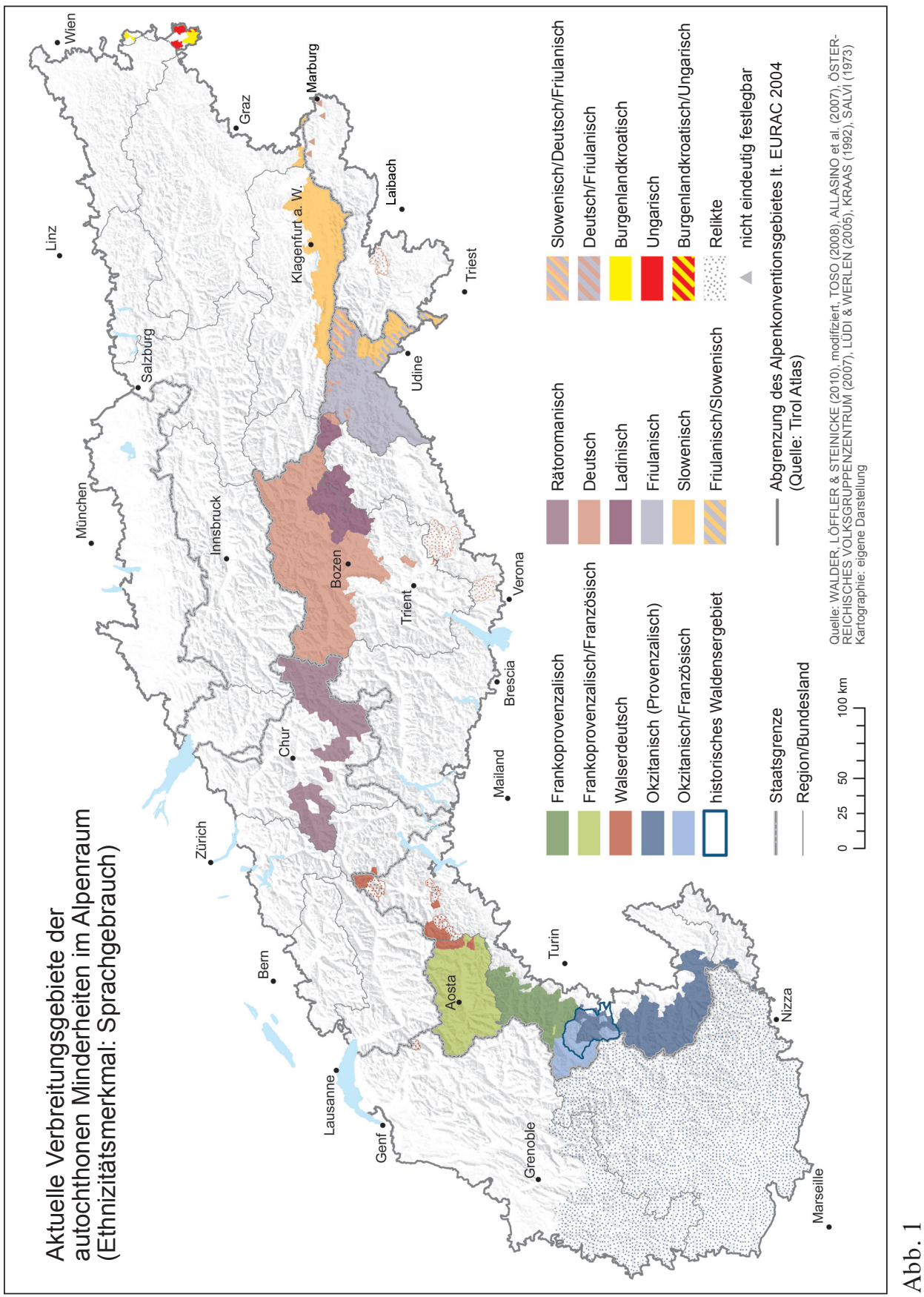




\subsection{Außengruppen (nationale Minderheiten)}

Diese haben ihr kulturpolitisches Zentrum im Ausland und können sich in einem gewissen Maß auf dessen politische, wirtschaftliche und kulturelle Kraft stützen. Im Alpenraum gilt das für die Slowenen in Friaul [Friuli] (mit Görz [Gorizia]), Kärnten und der Steiermark bzw. für die deutschsprachigen Südtiroler und Kanaltaler, ferner auch für die Kroaten und Ungarn im Burgenland. Nationale Minderheiten entstehen einerseits durch Grenzziehung und Grenzveränderungen, andererseits durch Auswanderung von Volksgruppen. Eine Ausnahmestellung nehmen hier die Frankoprovenzalen im Aostatal ein (vgl. BAuER 1999), die zu keiner Zeit zu Frankreich gehörten, jedoch von dessen kulturellem Einfluss durchaus profitieren. Eine weitere Einschränkung des genannten Begriffsinhaltes betrifft jene Minderheiten, deren Hauptgruppe zwar im Ausland siedelt, dort aber ebenfalls bloß eine Minderheit stellt oder dort nicht als Minderheit anerkannt ist. Dies ist bei den Okzitanen (Provenzalen) im Piemont [Piemonte] der Fall, die ihre (staatlich ungeschützte) Hauptgruppe in Frankreich haben.

Die Gebiete mit slowenischen Minderheiten in Österreich und Italien bilden den Rest eines einst viel weiter nach Westen und Norden reichenden slowenischen (oder alpenslawischen) Territoriums. Im gemischtsprachigen Teil Kärntens - einschließlich der Städte Klagenfurt am Wörthersee und Villach ${ }^{6}$ - bekennen sich heute knapp 5\% der Wohnbevölkerung als Slowenen (ohne Villach und Klagenfurt a.W. rund 10\%). Der jahrhundertealte Bevölkerungs- und Raumverlust der Kärntner Slowenen setzt sich bis in die Gegenwart fort. Er ist bereits mehrfach kartographisch veranschaulicht worden (GAmerith 1994, Steinicke 2001a).

Die steirischen Slowenen, die rechtlich gesehen den Kärntner Slowenen gleich stehen, sind eine der wenigen Volksgruppen Europas, die zahlenmäßig größer werden: Zwischen 1991 und 2001 ist diese Gruppe um rund 30\% gewachsen - von 1.695 auf 2.192 Personen. Der Grund dafür liegt zweifellos in einer Renaissance des ethnischen Bekenntnisses. Zuwanderungen aus Slowenien dürften in den 1990er Jahren - ungleich zur Zeit nach dem Zweiten Weltkrieg - kaum noch eine Rolle gespielt haben (UNKART 1997, ČEDE \& FLECK 2005, ÖSTERREICHISCHES VOLKSGRUPPENZENTRUM 2007). Abbildung 1 zeigt jedoch nur die steirischen Slowenen des Alpenraums.

Schon im 14. Jh. zog sich die „Slavia Veneta“ weitgehend ins Gebirge zurück. Heute umfassen die slowenischen Siedlungsgebiete in Friaul (mit Görz) das Resiatal [Valle di Resia] sowie die oberen Torre- und Natisonetäler mit den Collio-Slowenen von Görz. Zusammen mit den Kanaltaler Slowenen beträgt ihre Zahl rund 20 .000. Ihr territorialer Rückzug im vergangenen Jahrhundert wurde ebenfalls schon öfters dargestellt, wobei dafür im Unterschied zu den Kärntner Slowenen weniger die Assimilation als vielmehr die Entvölkerung die Hauptrolle spielte (STEInICKe 2002).

Die deutschsprachige Minderheit (ohne Sprachinseln) setzt sich aus den Südtirolern und Kanaltalern zusammen, deren Gebiete nach dem Ersten Weltkrieg an Italien

\footnotetext{
6) Abbildung 1 betrachtet diese zwei Städte als Teil des gemischtsprachigen Gebietes, zumal hier die meisten Kärntner Slowenen leben.
} 
kamen. ${ }^{7)}$ Eine europaweite Vorrangstellung in Sachen Minderheitenschutz nehmen aufgrund des Autonomiestatutes von 1947 und der zusätzlich anerkannten Rechte im Rahmen des „Paketes 1972“ die deutschsprachigen Südtiroler ein. Mit über 300.000 Angehörigen stellen sie zahlenmäßig die größte Minderheitengruppe des Alpenraums dar. Zudem bilden sie in ihrem Siedlungsgebiet die absolute Mehrheit, was zugleich bedeutet, dass die dort lebenden Italiener sozusagen eine „Minderheit im eigenen Staat" sind. Dies führt zum seltenen Fall, dass der staatstragende Bevölkerungsteil sich an die „Minderheit“ assimiliert. Eine wesentliche Rolle für diese Entwicklung spielt - ungleich zum Aostatal - das getrenntsprachliche Schulsystem. Außerdem ist für eine Anstellung im öffentlichen Dienst der Nachweis der erfolgreich bestandenen sogenannten Zweisprachigkeits- bzw. Dreisprachigkeitsprüfung (ladinische Sprachgruppe) zwingend vorgeschrieben, und die Vergabe der Stellen erfolgt nach dem ethnischen Proporz (vgl. Steininger 2003).

Im Aostatal, das 1948 ebenfalls den autonomen Status erhielt, fehlte es lange Zeit an spezifischen Maßnahmen zur Umsetzung der dort verankerten Schutzbestimmungen und speziell im Bildungsbereich vielfach an Französisch-Lehrern. Im privaten Bereich hat das Französische im Aostatal ohnehin nie eine große Rolle gespielt, was vor allem mit der historischen Entwicklung und der fehlenden territorialen Bindung an Frankreich zusammenhängt. Muttersprache der Aostataler ist vielmehr das Frankoprovenzalische, ein Sammelbegriff für all jene Dialekte, die sich weder dem Französischen (langues d'oïl) noch dem Okzitanischen oder Provenzalischen (langues d'oc) zuordnen lassen.

Die frankoprovenzalischen Dialekte, welche die Bewohner des Aostatals und der im Süden angrenzenden Gebiete wertfrei „Patois“ nennen, wurden bislang noch nicht kodifiziert, was den Erhalt derselben maßgeblich erschwert. Aufgrund des relativ guten Schutzes durch das Sonderstatut deckt sich das Verbreitungsgebiet der Frankoprovenzalen im Aostatal auch heute noch mit der administrativen Grenze der Region, während diese Sprachminderheit im Piemont sowohl zahlenmäßig als auch an Territorium verliert - genauso wie die okzitanische (provenzalische) und französische Gruppe (vgl. WALDER et al. 2010, S. 186).

Das okzitanische Sprachgebiet im Alpenraum liegt größtenteils in Frankreich. In östlicher Richtung reicht er über die Staatsgrenze und umfasst alle italienischen Westalpentäler südlich des Bassa Val di Susa und des Val Cenischia (Kremnitz 1975, S. 13f.). In den südlichen Gemeinden der Provinz Cuneo wird bereits der ligurische Einfluss bemerkbar (Toso 2008, S. 128).

Mitverantwortlich für die Ausbreitung der okzitanischen und französischen Sprache in Italien waren die Waldenser, eine religiöse Gemeinschaft, welche sich infolge der Inquisition unter anderem in den italienischen Westalpentälern Val Chisone, Val Germanasca und Val Pellice niederließen und sich im religiösen Bereich ausschließlich

\footnotetext{
7) Das Miestal [Mežica] und die Gemeinde Seeland [Jezersko], die nach dem Ersten Weltkrieg von Kärnten wegkamen und heute zu Slowenien gehören, werden nicht mehr im Zusammenhang mit deutschen Siedlungsgebieten in Slowenien erwähnt (Europarat 2010).
} 
des Französischen bedienten. Obwohl sich im Rahmen des Gesetzes Nr. 482/99 alle Kommunen des historischen Waldensergebietes als französischsprachig deklarierten, beschränkt sich der Anteil jener, die tatsächlich im alltäglichen Gebrauch neben dem Okzitanischen die französische Sprache verwenden, auf einige wenige Fraktionen. Anders verhält es sich hingegen in den Gemeinden des Alta Val di Susa und des Alta Val Chisone, wo aus historisch-administrativen Gründen der frankophone Einfluss über den Alpenhauptkamm herüberreicht. Dort erleichtert die unmittelbare Nähe zu Frankreich den Erhalt des Französischen, wobei Rück- und Zuwanderungen hauptsächlich als Folge von Intermarriage - dazu einen wesentlichen Beitrag leisten (Allasino et al. 2007, S. 46).

Ähnlich wie bei den steirischen Slowenen sind in Abbildung 1 auch bei den burgenländischen Kroaten und Ungarn nur die im Alpenraum liegenden Gemeinden berücksichtigt worden (kroatisch: Mattersburg, Stadtschlaining, Weiden bei Rechnitz und Markt Neuhodis; ungarisch: Bernstein, Lockenhaus und Markt Neuhodis).

\subsection{Eigengruppen (Domestic Minorities oder regionale Minderheiten)}

Ihr Siedlungsgebiet liegt nur in einem Staat und bildet zugleich das Kerngebiet ihrer Kultur auf der Erde. So sind die Rätoromanen, Friulaner und Ladiner für ihren Schutz letztlich auf sich allein angewiesen. Ihre Ethnogenese erfolgte an Ort und Stelle, wobei es nicht überrascht, dass bei ihnen die Territorialregression besonders ausgeprägt war.

Seit den Arbeiten des Romanisten Ascoli (1873) gelten die drei Gemeinschaften als eine eigene Gruppe, für die im deutschen Sprachraum die Begriffe „Ladiner“ (Ascoli 1873), ,Rätoromanen“ (GARTNER 1910) sowie „Alpenromanen“ (GAMILLSCHEGG 1936) in Verwendung gekommen sind. Ihr Verbreitungsgebiet, das weite Teile der Alpen und des Alpenvorlands umfasste, wurde von Norden und Nordwesten durch bajuwarischalemannische, von Osten durch slawische und von Süden und Südwesten durch oberitalienische Gruppen eingeengt, die zugleich das vorromanische und romanische Grundsubstrat veränderten. An die gemeinsamen ethnischen Wurzeln erinnern heute nur noch linguistische Parallelitäten, von einer großen „alpenromanischen Volksgruppe“ kann aber nicht die Rede sein.

Für die rund 60.000 im Alpenraum lebenden Friulaner bilden die Folgen der Abwanderungsperiode des 20. Jhs. - und hier in erster Linie die Überalterung - das größte ethnopolitische Problem. In der Montagna Friulana haben zudem Wohnsitzverlagerungen im Zuge des Wiederaufbaus nach der Erdbebenkatastrophe von 1976 dazu beigetragen, den massiven Einwohnerschwund in vielen friulanischen Tälern zu verstärken. Zahlreiche Ortswüstungen, wie sie vor allem im Canal del Ferro zu finden sind, waren die Folge (vgl. ČEDE \& STEINICKE 2007). Südfriaul hat hingegen durch den Wiederaufbau und die damit verbundenen finanziellen Zuwendungen die notwendigen Impulse für den wirtschaftlichen Aufschwung erhalten. Hier lebt auch der größte Teil der friulanischen Volksgruppe (ca. 450.000). 
Weniger von Venetien [Veneto] als vielmehr von Deutschtirol geprägt sind die rund 33.000 Dolomitenladiner. Ihr Siedlungsgebiet verteilt sich auf die drei Provinzen Südtirol, Trentino und Belluno rund um den Sellastock. Einen Sonderfall bilden ca. 6.000 Bewohner des Comelico südlich von Osttirol, die zwar Ladinisch sprechen, jedoch nie zu Tirol gehörten.

Dass die dreisprachigen Dolomitenladiner des Grödner- und des Gadertales in Südtirol in den letzten Dekaden sogar an Sprechern gewonnen haben, lässt auf eine Rückbesinnung und Rückwanderung in die Täler schließen, nicht zuletzt aufgrund der günstigen wirtschaftlichen Entwicklungen im Bereich des Tourismus. Seit 1972 gilt Ladinisch als dritte Amtssprache in Südtirol.

Wenngleich die Fassa-Ladiner nicht denselben Minderheitenschutz wie die Südtiroler Ladiner genießen, gewährt ihnen die Provinz Trient [Trento] weitgehende Rechte in den Bereichen Schule, Medien und Öffentlichkeit, und so konnten auch sie bis heute einen konstanten Siedlungsraum bewahren.

Die Ladiner der bellunesischen Gemeinden Livinallongo del Col di Lana (Buchenstein), Colle Santa Lucia und Cortina d'Ampezzo sowie des Comelico wurden hingegen bis zum Inkrafttreten des Gesetzes Nr. 482/99 nicht in den Minderheitenschutz einbezogen (Steinicke 2001b).

Mit 27.038 Personen (2000) sind die Graubündner Rätoromanen der Zahl nach kleiner als die Dolomitenladiner (LÜDI \& WERLEN 2005). Den jahrhundertelang anhaltenden Raumverlust dieser Schweizer Volksgruppe hat KRAAS (1992a) in ihrer Monographie veranschaulicht. Er ist inzwischen so weit fortgeschritten, dass der räumliche Zusammenhang innerhalb ihres Territoriums verloren ging. Zwischen 1990 und 2000 sank der Anteil der Bündneromanen an der Kantonsbevölkerung von 17,1 auf 14,5\%; im Jahr 1980 betrug er immerhin noch 22\%. Diese Einengung des Sprachgebietes erfolgt einerseits von Norden her durch eine fortschreitende Germanisierung, andererseits aber auch durch eine Berg-Talwanderung. Verschiedene Gegenden sind am Talboden nämlich rätoromanisch, in den Höhenlagen jedoch walserdeutsch besiedelt. Besonders auffallend sind dabei die Verhältnisse ganz im Westen des Minderheitenterritoriums, im rätoromanischen Bezirk Surselva, wo vereinzelt der deutsche Walserdialekt inselhaft dominiert - vor allem in Obersaxen.

\subsection{Deutsche Sprachinseln}

Bei Siedlungsgebieten deutschsprachiger Gruppen handelt es sich ausnahmslos um kleinräumige Refugien im Süden der Alpen, die in linguistischer Hinsicht Inseln oder Halbinseln (Sprachkeile) bilden. Gemeinsam ist auch ihre Entstehung. Die deutschen Sprachinseln in den West- und Ostalpen gehen auf den hoch- bis spätmittelalterlichen Siedlungsausbau zurück, als Walliser („Walser“), Tiroler und Kärntner Kolonisten südlich des geschlossenen deutschen Sprachgebiets angesiedelt wurden. Sowohl im Westen als auch im Osten hat allerdings das Romanische zahlreiche dieser entstandenen Enklaven überschichtet. 
Abbildung 1 zeigt ihr heutiges Verbreitungsgebiet. Es handelt sich dabei durchwegs um kleinere Ortschaften mit einigen hundert Einwohnern. Im Bereich der Westalpen sind dies die Walserkolonien der Provinzen Aosta, Verbano Cusio Ossola und Vercelli, wo rund 1.000 Personen die alemannische Mundart im Alltag verwenden. Dieses Idiom hat sich am stärksten in den drei Lystaler Gemeinden Gressoney-Saint-Jean, Gressoney-la-Trinité und Issime (Provinz Aosta/Aoste) gehalten. Auch in Alagna, Rimella (Provinz Vercelli), Formazza und Macugnaga (Provinz Verbano Cusio Ossola) sowie in Bosco Gurin im Schweizer Kanton Tessin trifft man auf alemannische Mundartsprecher, doch nimmt deren Zahl stetig ab.

Ähnliches gilt für die bairisch-österreichischen Sprach- und Sprachhalbinseln der Ostalpen, wo rund 2.800 Deutschsprachige leben. Der Großteil davon, ca. 1.000, entfällt auf Sappada/Bladen am Südfuß des Karnischen Hauptkamms (Provinz Belluno). Der Rest verteilt sich auf Timau/Tischelwang und Sauris/Zahre (Provinz Udine), auf die Sprachinseln im Fersental [Val dei Mòcheni] und auf Luserna/Lusern (Provinz Trient). In den vicentinischen Sette Comuni (Sieben Gemeinden) und den veronesischen Tredici Comuni (Dreizehn Gemeinden) sowie im slowenischen Sorica (Zarz) bestehen allerdings nur noch unbedeutende Sprachreste.

Eine ethnische Zuordnung, die bloß den objektiven (mutter-)sprachlichen Befund berücksichtigt, findet allerdings nicht von allen Seiten Zustimmung. Manche Autoren wie Francescato \& Solari Francescato (1994) lehnen die Bezeichnung „deutsche Sprachinseln“ überhaupt ab, da ihre einheimischen Bewohner, wenn nicht schon an die Staatssprache assimiliert, ausnahmslos zwei- oder dreisprachig seien. Sie schlagen stattdessen vor, von „mehrsprachigen Gemeinschaften“ zu sprechen.

Es sind jedoch weniger diese terminologischen Auseinandersetzungen als vielmehr Tendenzen einer „diffusen Ethnizität“, die im Süden der Alpen ein ethnogeographisches Problem bilden. Besonders in den deutschen Sprachinseln bestehen zum Teil erhebliche Ethnizitätsprobleme, was ihre ethnische Zuordnung in Frage stellt. Jedenfalls ist es nicht zweckmäßig, sie als ,nationale Minderheit“ zu betrachten. Diese Dimension wird im folgenden Kapitel näher ausgeführt.

\section{Subjektiver Befund}

Bisher war hauptsächlich von primordialen Ethnizitätsfaktoren - wie Sprache oder gemeinsame Geschichte - die Rede. Tatsächlich deckt sich im Alpenraum der objektive linguistisch-historische Sachverhalt nicht immer mit dem ethnischen Bekenntnis, Bewusstsein bzw. der Selbst- und Fremdeinschätzung. Dies kann einerseits im Zusammenhang mit Tendenzen einer ethnischen Selbstentfremdung (,diffuse Ethnizität“) zu sehen sein, andererseits lassen sich auch willkürliche Manipulationen aufzeigen. 


\subsection{Die kommunale Selbsteinschätzung}

Das in Kapitel 2 angesprochene Staatsgesetz Nr. 482 zum Schutz der „Minoranze linguistiche storiche“ räumt den italienischen Gemeinden das Recht ein, sich in ethnolinguistischer Hinsicht selbst zu deklarieren. Kommunen, in denen autochthone Minderheiten leben, können so in den Genuss von staatlichen Finanzmitteln kommen, um die kulturellen Besonderheiten zu bewahren.

Die allgemeine Unkenntnis über die exakte Verbreitung der Volksgruppen im italienischen Alpenraum macht es jedoch möglich, dass Gemeinden, in denen die Minderheitensprache schon seit Jahrhunderten verschwunden ist, sich selbst als Minderheitenterritorium ,einschätzen“. Aus diesem Grund erschien es notwendig, für den italienischen Alpenanteil die „Minoranze linguistiche storiche“ auch nach diesem Aspekt kartographisch darzustellen. Es ist keinesfalls überraschend, dass die Minderheitenterritorien bei der kommunalen Selbsteinschätzung (vgl. Abb. 2) flächenmäßig größer sind. Vergleicht man die Abbildungen 1 und 2, so unterliegen alle ethnolinguistischen Minderheiten außerhalb der autonomen Regionen Südtirol-Trentino und Aostatal solchen „territorialen Veränderungen“. Eine ausführliche Diskussion darüber findet sich bei WALDER et al. (2010). Im Folgenden sollen die zwischen den Abbildungen 1 und 2 hervortretenden Unterschiede am Beispiel der Dolomitenladiner diskutiert werden.

Auf Battisti (1941) geht die Vorstellung zurück, dass alle fünf ladinischen Täler um den Sellastock von den Altsiedlungsräumen im Eisack- und Pustertal aus besiedelt wurden, wo im beginnenden Hochmittelalter die rätoromanische Bevölkerung offensichtlich noch überwog. LuTz (1966, S. 55) modifizierte diese These dahingehend, dass durch die hochmittelalterliche Kolonisationstätigkeit eine bereits bestehende punkthafte rätoromanische Besiedlung flächenhaft ausgeweitet wurde. Scharfe Sprachgrenzen, zum Teil durch Schluchtstrecken oder Gebirgskämme verstärkt, sind nur dort zu finden, wo das Dolomitenladinische an das Deutsche grenzt. Im Süden hat der Sprachkontakt mit den Nachbarn hingegen eine Zone geschaffen, in der das ladinische Idiom allmählich in das Italienische bzw. Friulanische übergeht (vgl. Pellegrini 1977). Für die Süd- und Ostbegrenzung „Ladiniens“ ist deshalb die Zugehörigkeit zu Alttirol entscheidend. Eine Ausnahme bildet, wie erwähnt, das Comelico.

Es war ebenso bereits die Rede davon, dass die ladinischen Gebiete Südtirols und des Trentino im Rahmen der Provinzautonomien Minderheitenrechte besitzen, die Ladiner in der Buchensteiner Talschaft sowie in Cortina d'Ampezzo (Provinz Belluno) jedoch nicht. Durch die neue Gesetzeslage können die einzelnen italienischen Gemeinden nun seit dem Jahr 2000 einen aktiven Beitrag zum Erhalt bzw. zur Wiederbelebung der kulturellen und sprachlichen Vielfalt leisten. Der kommunale Beschluss, sich zu einer oder mehreren Sprachminderheiten zu bekennen, ist jedoch nicht selten lediglich mit der Hoffnung auf finanzielle Unterstützung zu erklären (Art. 5, 482/99). Durch die unklare linguistische Südgrenze Ladiniens ist es nur verständlich, dass die Gemeinden im Agordino und Cadore nun auch die Vorteile des neuen Gesetzes nützen und sich dementsprechend genauso als ladinisches Minderheitengebiet deklarierten (vgl. Abb. 2 und 3). 
Somit hat die neue Gesetzeslage eine seit Mitte der 1970er Jahre in der Region Venetien erkennbare „neoladinische Bewegung“ (vgl. GoEBL 1997) verstärkt. Als Folge verlieren die „Tiroler Dolomitenladiner“ von Cortina d“Ampezzo, Colle Santa Lucia und Livinallongo allmählich ihre Sonderstellung innerhalb Venetiens. Finanzielle Unterstützung für den Minderheitenschutz verlangen daher nicht mehr bloß diese drei Gemeinden allein, sondern eine unübersehbare Fülle von Kommunen des Veneto. Es ist deshalb nicht verwunderlich, dass sich im Oktober 2007 fast 80\% der Bewohner der Buchensteiner Talschaft und Cortinas für den Übergang an die Provinz Bozen (Südtirol) ausgesprochen haben.

\section{2 „Diffuse Ethnizität"“}

Es ist Schnittpunkten von Kulturen eigentümlich, dass es leicht zu Rivalitäten, Auseinandersetzungen und sogar offener Feindschaft kommen kann, aber auch zur Symbiose und zum gegenseitigen kulturellen Austausch. Das jahrhundertelange Zusammenleben im Gebirge hat jedenfalls Lebensgewohnheiten und Wertvorstellungen entscheidend geprägt. So stößt man in den Minderheitsgebieten auf unterschiedliche Vorstellungen über die eigene Ethnizität, was die ethnische Zuordnung der verschiedenen sprachlichen Gemeinschaften oftmals erheblich erschwert. Dies fällt besonders in Friaul auf, ferner aber auch in Südkärnten, im Aostatal und bei den Provenzalen in den französischen Westalpen, wo sich Eigenarten im ethnischen Bekenntnis entwickelt haben („diffuse Ethnizität“, STEINICKE 1991, S. 178-185).

So finden wir Slowenen, die sich als Deutsche (in Kärnten und zum Teil im Kanaltal [Valcanale]), manchmal auch als Russen (sic! im Resiatal) bekennen; Friulaner als Italiener im ethnischen Sinn oder auch als Dolomitenladiner (in Erto); Sprachinseldeutsche, die sich als eigene ethnische Gruppe sehen; Italiener, die eine walserdeutsche und Provenzalen, die eine französische ethnische Identität haben. Im Folgenden seien nur einige Ethnizitätsprobleme näher ausgeführt.

Ein Gutteil der Kärntner Slowenen bekennt sich nicht zum slowenischen, sondern zum deutschen Kulturraum und lehnt es ab, den Slowenen zugerechnet zu werden. Für diese Bevölkerungsgruppe, die auch nicht die für den Minderheitenschutz vorgesehenen Möglichkeiten in Anspruch nimmt, hat sich der Ausdruck „Windische“ eingebürgert (vgl. dazu UnKarT et al. 1984, S. 41-43). Daher ist das für die Kärntner Slowenen in Volkszählungen ausgewiesene Zahlenmaterial höchst problematisch. Zweifellos lässt sich in Südkärnten eine ethnische Rückbesinnung beim gut ausgebildeten Bevölkerungsteil erkennen, doch zeigen die amtlichen Spracherhebungen, dass die Zahl der Slowenen weiterhin rückläufig ist. Untersuchungen ergaben, dass knapp 50.000 Kärntner zu Hause Slowenisch sprechen. Der Zensus gibt allerdings nur ein knappes Viertel dieser Zahl wieder (STEINICKE \& ZupanČIČ 1994, 1995; STEINICKe 2001a).

Auch bei den Kanaltaler Slowenen sind unterschiedliche Vorstellungen zur ethnischen Identität vorhanden. Manche bezeichnen sich, wie die Kärntner Slowenen, noch immer als Windische, die meisten aber sehen ihre Ethnizität einfach als Kanaltaler (Vavti 2005, Vavti \& Steinicke 2006). 


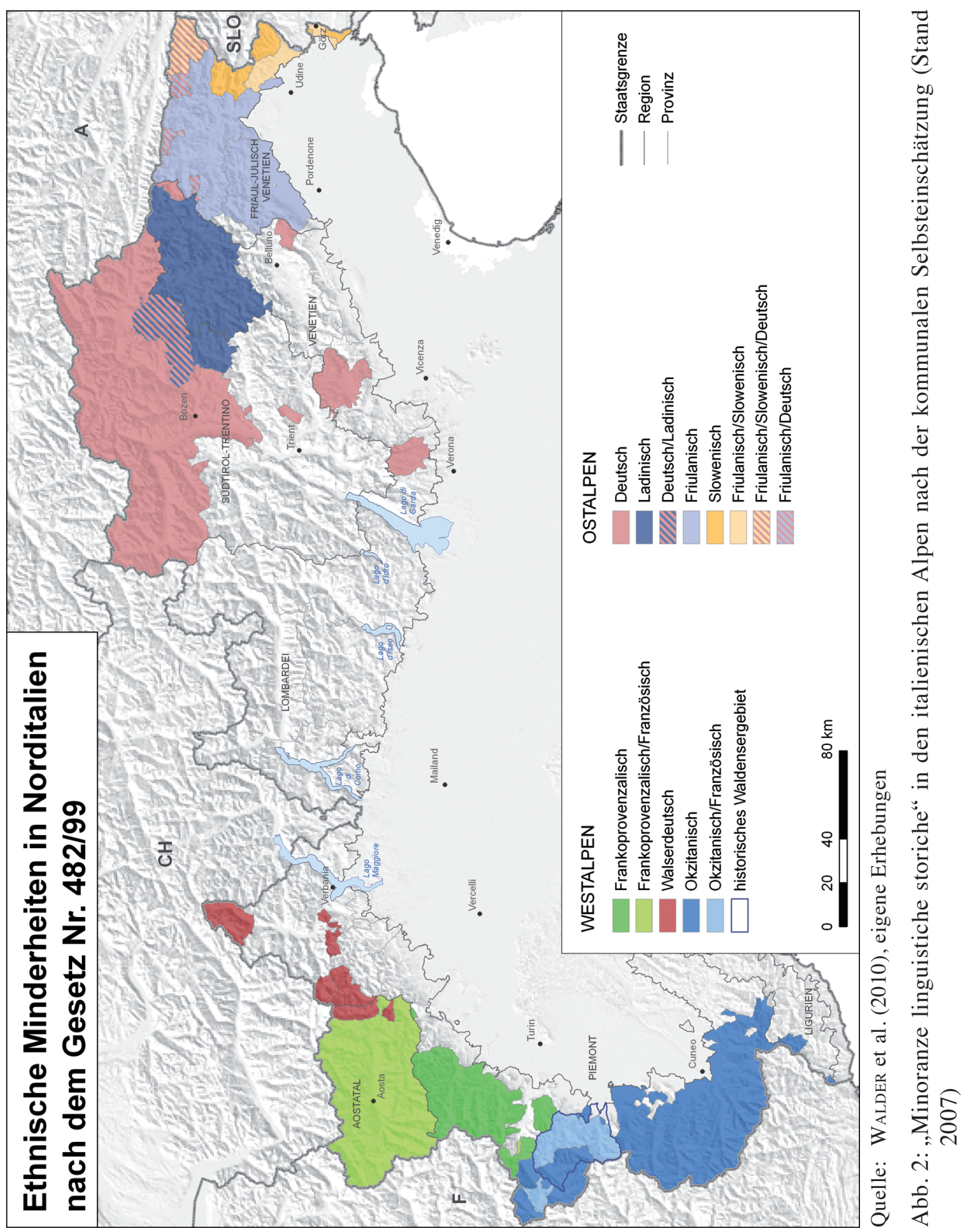




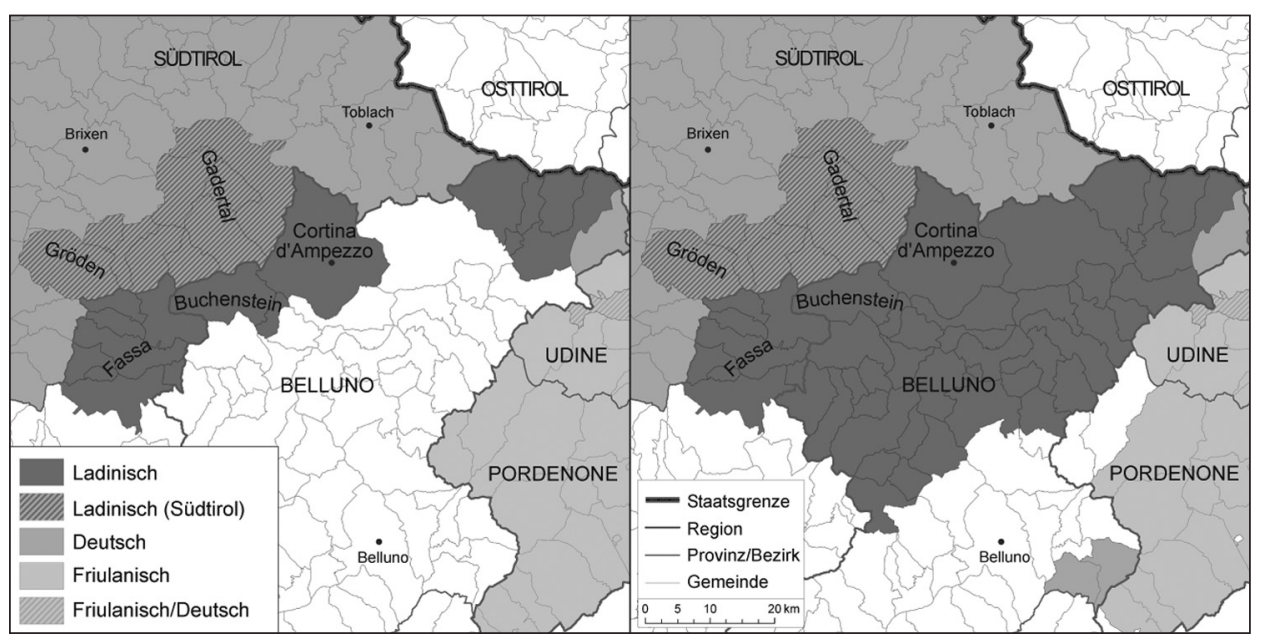

Quelle: Eigene Erhebungen (Stand 2007)

Abb. 3: Die Dolomitenladiner: traditionelles Siedlungsgebiet (links) versus Selbsteinschätzung der Gemeinden nach dem Staatsgesetz Nr. 482/99 (rechts)

Zwei Talschaften südlicher, im Resiatal, stößt man in Gesprächen mit Einheimischen nicht selten auf die Meinung, dass die Bewohner in sprachlicher Hinsicht entweder Russen seien oder Angehörige einer völlig eigenen Volksgruppe. Der besonders altertümliche Lautstand des dort gesprochenen Slowenischen („Rozajansko“) hat schon sehr früh slawischen Wissenschaftern und Dichtern Anlass geboten, das Resiatal zu besuchen (STEINICKE 1991, S. 161). Nachhaltigen Einfluss auf die ethnische Identität übten offensichtlich russische und ukrainische Linguisten des 18. und 19. Jhs. aus. BECKER (1971), der zu Beginn der 1970er Jahre eine Kartendarstellung über die Volksgruppen in den Ostalpen vorlegte, musste das Resiatal als „weißen Fleck“ belassen. Die befragte Gemeinde war sich nämlich nicht einig, welche slawische Sprache in ihrem Tal gesprochen werde.

Ähnlich sind die Verhältnisse auch bei den Sprachinseldeutschen in den Alpen. Viele Gespräche mit Einheimischen in den beiden Sprachinseln Friauls, Timau/Tischelwang und Sauris/Zahre, wiesen darauf hin, dass sie sich als Italiener fühlen, die in ihren Ortschaften einen für Auswärtige unverständlichen Dialekt sprechen. Durch das jahrhundertelange Zusammenleben der verschiedenen Volksgruppen schlägt sich die ethnische Identität der Sprachinseldeutschen in der Bezogenheit zum eigenen Dorf nieder, nicht jedoch in der Einschätzung, zum deutschen Kulturkreis zu gehören oder Vertreter der deutschen Kultur in Italien zu sein.

Obwohl immer wieder Deutschkurse in den Sprachinseln stattfinden, die zumeist von Österreich aus organisiert werden, bleibt sowohl im lokalen literarischen Schaffen als auch in neueren Dorfzeitungen und wissenschaftlichen Publikationen der alte deutsche Dialekt in italienischer oder in selbst geschaffener Transkription das geschriebene Wort (vgl. Abb. 4). 


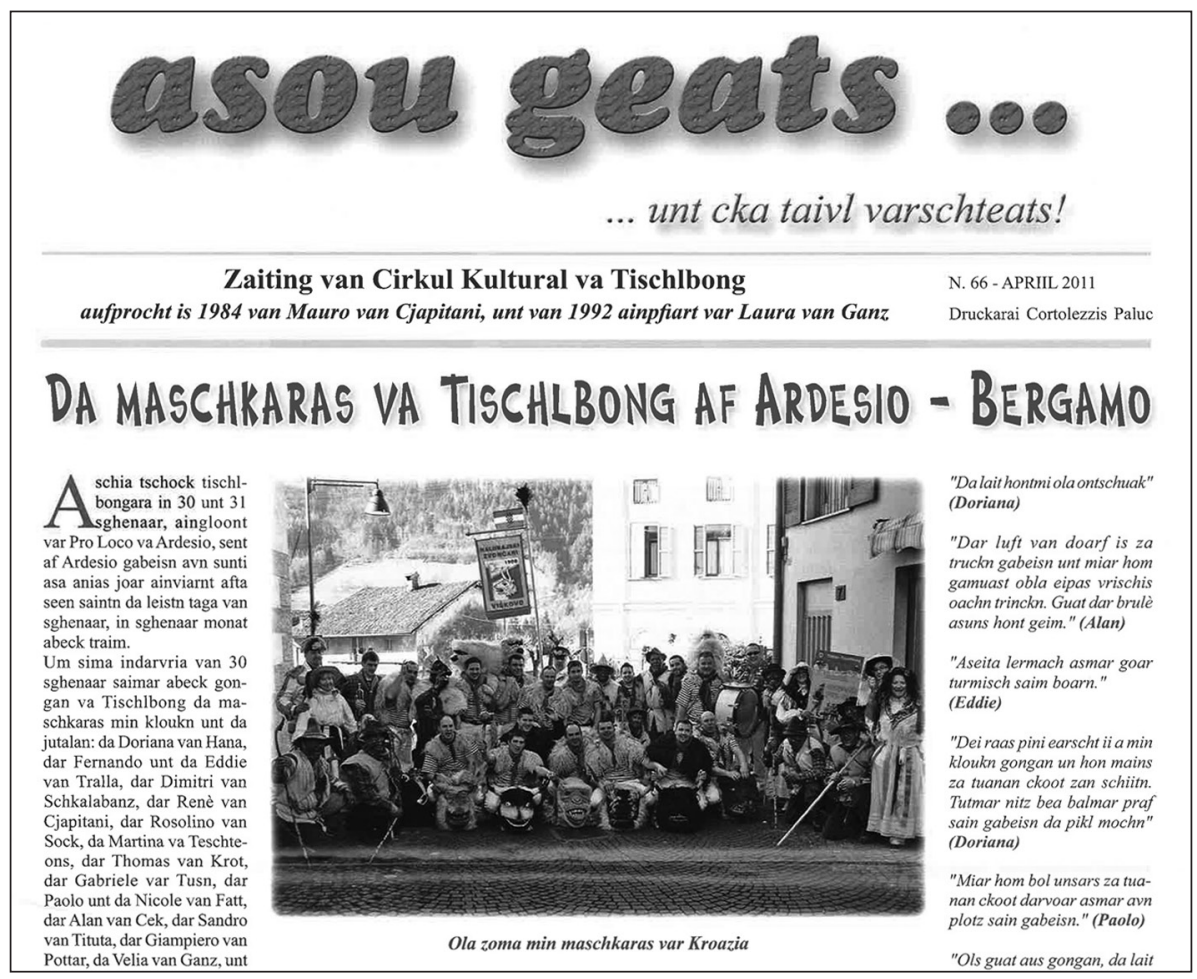

Abb. 4: Die Dorfzeitung in Timau/Tischelwang („Tischlbong“) „a sou geats ... und cka taivl varschteats!“ in selbst kodifizierter Sprache

Trotz des Wissens um die ethnische Herkunft wenden sich zum Beispiel die Akteure im Tischelwanger Kulturkreis demonstrativ gegen die Zugehörigkeit zum deutschen Sprachraum und lehnen dementsprechend die Verwendung des Standarddeutschen ab. Man gewinnt den Eindruck, dass die vereinzelt auftauchenden hochdeutschen Bezeichnungen eher Dankbarkeit für österreichische Hilfe bei Wahrung kulturspezifischer Interessen als Bewusstsein der Deutschsprachigkeit ausdrücken.

In einer modifizierten Form gilt dies ebenso für die deutschen Sprachinseln der Westalpen (STEINICKE \& PIOK 2003). Besonders in den Walser-Sprachinseln im Aostatal ist die mittelalterliche Herkunft aus dem Schweizer Wallis und damit das Walsertum bei den Bewohnern noch stark verankert. Im Gegensatz zu den deutschen Sprachinseln in Friaul (Ausnahme: Sappada/Bladen) wird hier auch die ethnische Sonderstellung bewusst im Tourismus eingesetzt. Gezielt wirbt man damit, eine Walsergemeinde zu sein, und Vieles ist danach ausgerichtet, sich als eine kulturgeschichtliche Besonderheit zu zeigen (Namengut, Kuckucksuhren, Baustil). Zahlreiche italienische Zuwanderer deklarieren sich als Walser - obwohl sie das „Titsch“ oder „Töitschu“ (oftmals selbst in Ansätzen) nicht verstehen. Sie entscheiden sich vom Hausnamen bis zu den Vornamen ihrer Kinder für die deutsche Walser-Variante. Insgesamt bekennen sich mehr 


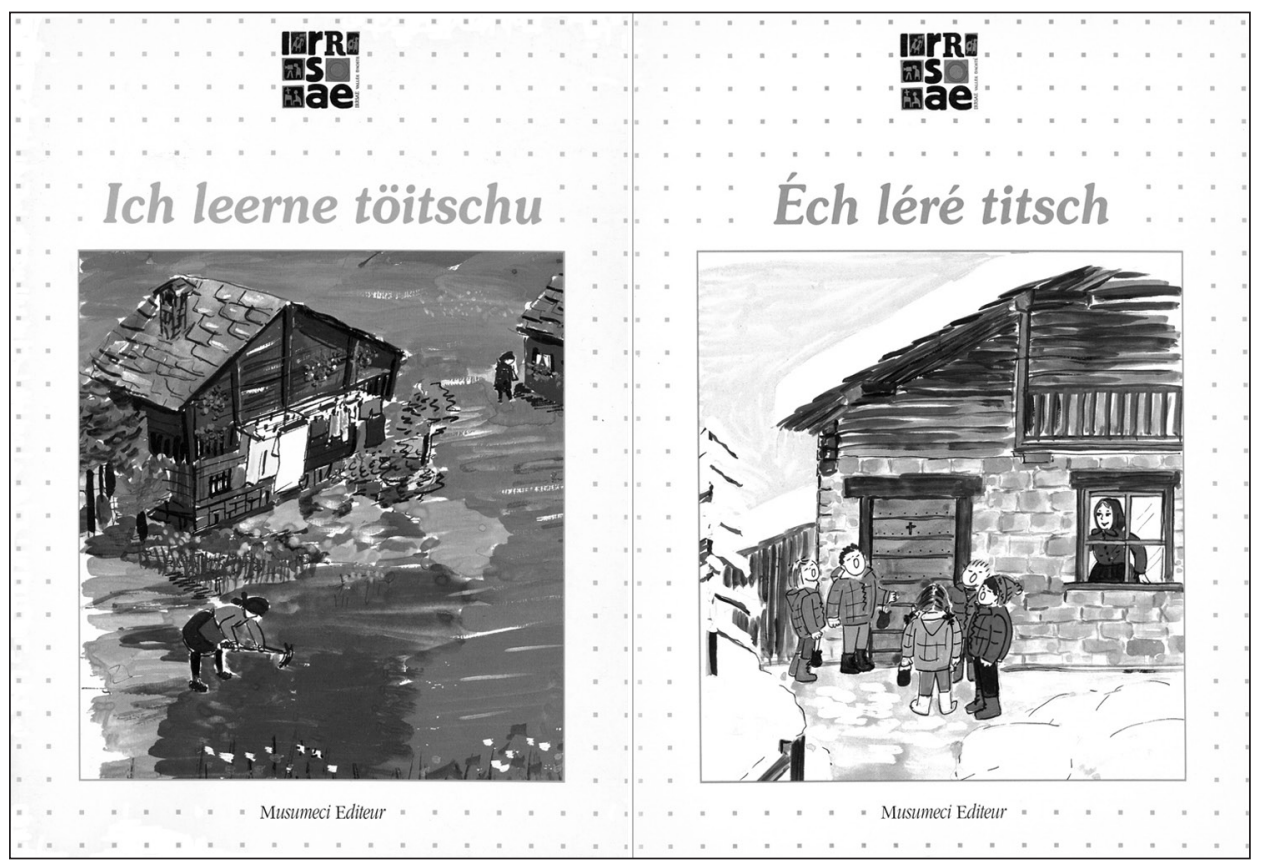

Abb. 5: Kinder-Lehrbücher des Walserdeutschen im Aostatal: Issime (links), Gressoney (rechts)

als doppelt so viel Einwohner als Walser als es dort überhaupt Deutschsprachige gibt.

Im Aostatal, wo für die Walserdeutschen schon seit längerer Zeit offizielle Schutzmaßnahmen bestehen, zeigt sich aber ein ähnliches Phänomen wie in den Sprachinseln in Friaul: die Ablehnung der deutschen Schriftsprache und die alleinige Weitergabe des Dorfdialekts. So entwickelte man in Issime und Gressoney, zwei Dörfern, die 7 km voneinander entfernt sind, jeweils ein eigenes Kinder-Lehrbuch des Dorfdialektes (vgl. Abb. 5).

Der Zeitgeist der letzten beiden Jahrzehnte unterstützt und verstärkt eine solche Entwicklung. Die im Zusammenhang mit der Globalisierung zu sehende Auflösung nationalstaatlicher Wirtschaftsräume führt zu Gegenbewegungen, die sich unter anderem im Hervorheben des Regionalen und Lokalen bemerkbar machen. Kulturelle Singularitäten gewinnen in der Gegenwart an Bedeutung, und dementsprechend sehen sich die Mitglieder kleiner ethnolinguistischer Gruppen in zunehmendem Maße als Bereicherung einer Region. Obwohl dadurch die Bezogenheit zum jeweiligen Dorf und das Lokalbewusstsein wachsen, können gewisse Auswirkungen dieser besonderen Selbsteinschätzung - und hier vor allem die Ablehnung der Standardsprache -, wie es beispielsweise in den deutschen Sprachinseln und im slowenischen Resiatal der Fall ist, den Erhalt der ethnischen Vielfalt auch gefährden. 


\section{Bedrohung des ethnischen Mosaiks und Perspektiven}

Obwohl inzwischen - abgesehen vom Sonderfall Frankreich - allen autochthonen Minderheiten in den Alpen besondere Rechte zum Schutz ihrer kulturellen Eigenarten zustehen, kann ihre fortschreitende Assimilierung an das jeweilige staatstragende Volk nicht bestritten werden. Dabei spielt die „diffuse Ethnizität“" keine unbedeutende Rolle. Der Rückgang der ethnischen Diversität steht zugleich auch eng in Zusammenhang mit der demographischen Entwicklung. Selbst wenn vereinzelt Minderheitenterritorien zu wirtschaftlichen Aktivräumen zählen, vor allem Südtirol und touristisch geprägte Hochtäler sowie einige Teile des Aostatals, bilden sie in ihrer Gesamtheit in sozioökonomischer Hinsicht Problemregionen.

Insbesondere in jenen alpinen Gebieten, die durch naturräumliche und agrarsoziale Ungunstfaktoren gekennzeichnet sind, kam es bis weit in die 1970er Jahre aufgrund von mangelnden Beschäftigungsmöglichkeiten im sekundären und tertiären Sektor zu einer massiven Entvölkerung. Dies betraf vor allem Teile der slowenischen, italienischen und französischen Alpen. Wenngleich sich dort überall seit den 1980er Jahren die Bevölkerungsverluste abschwächen, fallen die Auswirkungen der aus der Abwanderungsperiode resultierenden ungünstigen biodemographischen Faktoren - vor allem der Überalterung - noch immer auf (LöfFler \& STEINICKe 2007, S. 214f.).

Zwar konnten sich die Minderheitensprachen speziell in peripheren und strukturschwachen Räumen halten, doch ist es unverkennbar, dass sie heute - außerhalb von Südtirol und in abgeschwächtem Maße des Aostatals - zum Großteil nur noch von Älteren beherrscht werden.

Rückläufige Einwohnerzahlen bringen den verschiedenen Volksgruppen zwangsläufig hohe Verluste, die umso schwerer wiegen, je kleiner die Minderheit ist. Die Minorisierung einer sprachlichen Minderheit in ihrem eigenen Siedlungsgebiet, wie sie bereits KREMNITZ (1975, S. 20) beschreibt, erfolgt aber nicht allein durch Abwanderung von Angehörigen derselben. Gleichermaßen schwächen Zuwanderungen und die damit verbundene verstärkte Interaktion mit der Mehrheitsbevölkerung, insbesondere durch „Intermarriage“, die Minderheiten.

In seiner Analyse belegte BeISMAnN (2009), dass die italienischen Alpen der demographischen Entwicklung in den französischen Westalpen gefolgt sind und es daher heute unrichtig sei, dort von ,großen Entsiedlungsgebieten“ (B̈̈TZING 2005, S. 288) zu sprechen. Wie im Rahmen des laufenden FWF-Forschungsprojektes von Innsbrucker Geographen bereits mehrfach aufgezeigt wurde (z.B. WALDER et al. 2010, S. 182f.), bilden auch die italienischen Alpengemeinden seit 2002 in ihrer Gesamtheit ein Zuwanderungsgebiet, und die überwiegende Mehrheit der Gemeinden tritt bereits mit einer wachsenden Einwohnerzahl hervor. Selbst in den besonderen demographischen Problemgebieten Nordfriaul und Cottische Alpen [Alpe Cottiche], wo das Geburtendefizit noch immer zu groß ist, als dass es vom positiven Wanderungssaldo statistisch aufgefangen werden könnte, ist ein signifikantes Abklingen der Entvölkerung feststellbar.

Im Zusammenhang mit der angesprochenen demographischen Trendwende in den italienischen Alpen spielt die „Amenity (-led) migration“ (Moss et al. 2009) eine beachtliche Rolle. Dieses Phänomen beschreibt die Verlagerung der Wohnsitzpräferenz vom urbanen Raum in abgelegene, aber attraktive ländliche (Gebirgs-)Regionen. Es 
ist neben den Sub- und Exurbanisierungsprozessen am Alpenrand sowie der Arbeiterrückwanderung (Remigration) die treibende Kraft der gegenwärtigen Siedlungserweiterung und des aktuellen Bevölkerungszuwachses in zahlreichen italienischen Alpengemeinden. Wochenend- und Freizeitwohnsitze werden immer öfter zu Zweit- oder Alterswohnsitzen, wodurch sich die Aufenthaltsdauer im Zielgebiet deutlich erhöht. Da immer mehr Personen arbeitsplatzungebunden sind, steigt die Motivation, auch berufliche Aspekte in den „neuen“ Wohnort zu verlagern.

Im Hinblick auf den Fortbestand der autochthonen Minderheiten verändert dieser neue demographische Prozess - außerhalb Südtirols, wo die Zuwanderung sehr restriktiv gehandhabt wird, - die ethnischen Proportionen und verstärkt letztendlich die italienischsprachige Majorität.

Darüber hinaus hat in vielen Gemeinden des Untersuchungsgebietes die „diffuse Ethnizität" einen hohen Grad erreicht.

Assimilation und demographischer Wandel sind also die beiden Kräfte, die den Bestand der bodenständigen Minderheiten bedrohen. Heute sind im gesamten Alpenraum die sozialen Spannungen aufgrund der Ethnie relativ gering, sieht man von ihrem gelegentlichen Aufflackern wegen toponomastischer Probleme sowie anderer ethnopolitisch aufgeladener Symbole in Südkärnten und Südtirol ab.

Bei den kleinen Minderheiten ist das Protestpotenzial aus biologischen Gründen reduziert - zumeist sind die Jungen bereits an die Majorität assimiliert. Das Konfliktpotenzial ist auch deshalb so gering geworden, weil es seit dem Jahr 2000 - mit Ausnahme der französischen Gebiete - keine ungeschützten Minoritäten mehr in den Alpen gibt.

So ist für alle Minderheitengebiete in den nächsten 15 Jahren ein Status-quo-Szenario am wahrscheinlichsten: Setzt sich der bisherige Trend fort, so verringert sich weiterhin der Bestand der ethnolinguistischen Minoritäten (Ausnahme: Südtirol). Die Minderheitensprache ist vornehmlich im älteren Bevölkerungsteil verankert. Durch seinen hohen Anteil an der Einwohnerzahl erscheint momentan der Erhalt der kleineren Ethnien nicht gefährdet zu sein. Es darf jedoch nicht außer Acht gelassen werden, dass sich mit dem Absterben der älteren Jahrgänge die Ethnodiversität reduziert.

Eine Variante zum genannten Szenario ist jedoch durchaus möglich: Ähnlich wie in den französischen und italienischen Alpen bereits feststellbar, ist zu erwarten, dass die Zahl der Zuwanderer auch in den übrigen Alpengebieten ansteigen wird. Angesichts der fortgeschrittenen Kommunikationstechnologie werden Personen aus den metropolitanen Räumen am Rande der Alpen ebenso (abgelegene) Alpentäler als neuen Wohnort oder als Zweitwohnsitz interessant finden. Sind dort die behördlichen Auflagen für eine Zuwanderung überwindbar, so dürfte die Einwohnerzahl künftig signifikant ansteigen.

Als weitere „Newcomers“ sind ferner Immigranten oder Flüchtlinge aus anderen Kulturkreisen zu nennen. Da in den genannten Peripherräumen günstiger Wohnraum vorhanden ist, hat diese Variante ebenso hohe Wahrscheinlichkeit. Die Entvölkerung des Gebirges würde dadurch zwar gebremst, der Erhalt der autochthonen ethnischen Struktur bliebe aber unverändert bedroht, wenngleich die Alpen durch die Immigration von Südosteuropäern, Türken, Tschetschenen, Moldauern oder Nordafrikanern noch „multikultureller“" würden. 


\section{Literaturverzeichnis}

AkademiJa nauk SSSR (Hrsg.) (1964), Atlas narodov mira [Atlas der Völker der Erde]. Moskva.

Allardt E. (1979), Implications of the Ethnic Revival in Modern Industrialized Society. A Comparative Study of the Linguistic Minorities in Western Europe. Helsinki, Societas Scientarium Fennica.

Allasino E., Ferrier C., Scamuzzi S., Telmon T. (2007), Le Lingue del Piemonte. In: IRES PIEmonte (Hrsg.), Quaderni di ricerca, 113. Torino, Istituto di Ricerche Economico Sociali del Piemonte.

Anderwald K., Krapf P., Smrtnik V. (Hrsg.) (1997), Sprachminderheiten: Herausforderung und Chance (= Kärnten Dokumentation, 15). Klagenfurt, Land Kärnten.

Ascoli G.I. (1873), Saggi ladini. In: Archivio Glottologico, 1, S. 1-556.

ASTAT - LANDESinstitut FÜR StATISTIK (Hrsg.) (2002), Volkszählung 2001. In: astat info, 17. Bozen.

BäTZING W. ( ${ }^{32005), ~ D i e ~ A l p e n . ~ G e s c h i c h t e ~ u n d ~ Z u k u n f t ~ e i n e r ~ e u r o p a ̈ i s c h e n ~ K u l t u r l a n d s c h a f t . ~}$ München, C.H. Beck.

B Aтtisti C. (1941), Storia linguistica e nazionale delle valli dolomitiche atesine. In: Archivio per l'Alto Adige, 36, S. 5-298.

BAUER R. (1999), Sprachsoziologische Studien zur Mehrsprachigkeit im Aostatal. Mit besonderer Berücksichtigung der Sprachgeschichte (= Beihefte z. Zeitschrift f. romanische Philologie, 296). Tübingen, Niemeyer.

BECKER H. (1971), Die Volksgruppen der italienischen Ostalpen. Begleitworte zum Versuch einer Kartendarstellung. In: Forschung zur allgemeinen und regionalen Geographie. Sonderband d. Kölner Geogr. Arbeiten (= Festschrift f. K. Kayser), S. 256-270. Köln, Steiner.

Beismann M. (2009), Aktueller demographischer Wandel in den italienischen Alpen. Unveröff. Dipl.-Arb., Univ. Innsbruck.

Berschin H., Felixberger J., Goebl H. (1978), Französische Sprachgeschichte. München, Hueber.

ČEDE P., FLECK D. (2005), Die steirischen Slowenen im Spiegel der amtlichen Volkszählungen. In: Europa Ethnica, 3-4, S. 101-114.

Čede P., Steinicke E. (2007), Ghosttowns in den Ostalpen. Das Phänomen der Entvölkerung im friulanischen Berggebiet. In: Geographica Helvetiva, 62, S. 93-103.

EURAC (2004), Definition des Perimeters der Alpenkonvention. Bozen.

EuRoparat (2010), ECRML - Europäische Charta der Regional- oder Minderheitensprachen: Europarat Empfehlungen zur deutschen Sprache in Slowenien. - http://www.agdm.fuen. org/doc/COE/SI.doc (Mai 2011).

Francescato G., Solari Francescato P. (1994), Timau. Tre lingue per un paese. Lecce, Congedo.

Gamerith W. (1994), Ethnizität und ihr zeitlich-räumlicher Wandel. Das Beispiel der Kärntner Slowenen (= Klagenfurter Geogr. Hefte, 12). Klagenfurt, Inst. f. Geogr. u. Regionalforschung.

Gamillschegg E. (1936), Allgemeine Bevölkerungsentwicklung Friauls, insbesondere das Friulanertum. In: Handwörterbuch d. Grenz- u. Auslanddeutschtums, 2, S. 588-589. Breslau, Ferdinand Hirt.

Gartner C. (1910), Handbuch der rätoromanischen Sprache und Literatur. Heidelberg, Niemeyer.

GazZetta ufficiale n. 297 del 20 dicembre 1999: Legge 15 dicembre 1999, n. 482. Norme in materia di tutela delle minoranze linguistiche storiche. Roma.

Goebl H. (1997), Der Neoladinitätskurs in der Provinz Belluno. In: Ladinia, 21, S. 5-57.

Heckmann F. (1992), Ethnische Minderheiten, Volk und Nation. Soziologie inter-ethnischer Beziehungen. Stuttgart, Enke.

Heller W. (2007), Ethnizität - Globalisierung - Transformation - Südosteuropa. Einführung in die Thematik. In: Heller W., Becker J., Belina B., Lindner W. (Hrsg.), Ethnizität in der 
Globalisierung. Zum Bedeutungswandel ethnischer Kategorien in Transformationsländern Südosteuropas (= Südosteuropa-Studien, 74), S. 11-28, München, Südosteuropa-Ges. Hutchinson J., Sмiтh A. (Hrsg.) (1996), Ethnicity. Oxford, Oxford Univ. Press.

JoRDAN P. (2002), Die ethnische Struktur des mittleren und östlichen Europas (anhand der Karten des Atlasses Ost- und Südosteuropa). In: KraAs F., Stadelbauer J. (Hrsg.), Nationalitäten und Minderheiten in Mittel- und Osteuropa (= Ethnos, 60), S. 24-50. Wien.

Karner S. (2001), Die deutschsprachige Volksgruppe in Slowenien und AVNOJ. In: Europ. Rundschau, 1, S. 25-32.

Kattenbusch D. (1997), Sprachkontakte in Südeuropa. In: Goebl H. (Hrsg.), Kontaktlinguistik: ein internationales Handbuch zeitgenössischer Forschung, S. 1318-1330. Berlin, de Gruyter.

KLoss H. (1969), Grundfragen der Ethnopolitik im 20. Jahrhundert (= Ethnos, 7). Wien, Braumüller.

KraAs F. (1992a), Die Rätoromanen Graubündens. Stuttgart, Steiner.

KraAs F. (1992b), Ethnolinguistische Bevölkerungsgruppen und Minoritäten als Aufgabe geographischer Forschung. In: Die Erde, 123, S. 177-190.

Kraas F., Stadelbauer J. (Hrsg.) (2002), Nationalitäten und Minderheiten in Mittel- und Osteuropa (= Ethnos, 60). Wien, Braumüller.

KraAs-Schneider F. (1989), Bevölkerungsgruppe und Minorität. Handbuch der ethnischen, sprachlichen und religiösen Bevölkerungsgruppen der Welt. Stuttgart - Wiesbaden, Steiner.

Kremnitz G. (1975), Die ethnischen Minderheiten Frankreichs - Bilanz und Möglichkeiten für den Französischunterricht (= Tübinger Beiträge z. Linguistik, 55). Tübingen, Narr.

Löffler R., Steinicke E. (2007), Amentiy Migration: Die neuen Bewohner der Alpen. Skizze eines Forschungsprojekts. In: Innsbrucker Geographische Gesellschaft (Hrsg.), Alpine Kulturlandschaft im Wandel. Hugo Penz zum 65. Geburtstag, S. 213-225. Innsbruck.

LÜDI G., WerLen I. (2005), Eidgenössische Volkszählung 2000. Sprachlandschaft in der Schweiz. Neuchâtel, BA f. Statistik.

Lutz W. (1966), Gröden. Landschaft, Siedlung und Wirtschaft eines Dolomitenhochtales (= Tiroler Wirtschaftsstudien, 21). Innsbruck, Wagner.

Moseley C. (Hrsg.) ( $\left.{ }^{3} 2010\right)$, Atlas of the World's Languages in Danger. Paris, UNESCO Publishing. - http://www.unesco.org/culture/en/endangeredlanguages/atlas (April 2011).

Moss L.A.G., Glorioso R., Krause A. (Hrsg.) (2009), Understanding and Managing Amenityled Migration in Mountain Regions. Proceedings of the Mountain Culture at the Banff Centre conference held May 15-19, 2008. Banff, Banff Centre Press.

Österreichisches Volksgruppenzentrum (2007), 2. Bericht zur Durchführung des Europäischen Rahmenübereinkommens zum Schutz nationaler Minderheiten in der Republik Österreich. Wien.-http://www.minelres.lv/reports/austria/NGOBerichtII2007deutsch.pdf (Mai 2011).

Opitz M. (2007), Die Minderheitenpolitik der Europäischen Union. Probleme, Potenziale, Perspektiven. Berlin, Lit Verlag.

Orioles V. (2003), Le minoranze linguistiche. Profili sociolinguistici e quadro dei documenti di tutela. Roma, Il Calamo.

Pan C.,Pfeil B.S. (2000), Die Volksgruppen in Europa.Ein Handbuch (=Ethnos, 56). Wien, Braumüller.

Pellegrini G.B. (1977), Carta dei dialetti d'Italia, I-II. Profile dei dialetti italiani. In: Centro Studio Dialettologia Italiana, 5. Pisa, Pacini.

Plasseraud Y. (1998), Les minorités. Paris, Montchrestien.

Rougier H., Sanguin A.L., Schwabe E. (1984), Frontières et minorités. In: Les Alpes (25 Congrès International de Géographie), S. 263-280. Grenoble, Univ. Press Grenoble.

SAllanz J. (2007), Bedeutungswandel von Ethnizität unter dem Einfluss von Globalisierung. Die rumänische Dobrudscha als Beispiel (= Potsdamer Geogr. Forschungen, 26). Potsdam, Univ.-Verlag Potsdam.

SALVI S. (1975), Le lingue tagliate. Storia delle minoranze linguistiche in Italia. Milano, Rizzoli. 
SAnguin A.-L. (1993), Quelle minorités pour quels territoirs? In: SAnguin A.-L. (Hrsg.), Les minorités ethniques en Europe, S. 5-18. Paris, L'Harmattan.

Spiss R. (Hrsg.) (2001), Die Fahrenden: „Innen- und Außenansichten“- Die Jenischen zwischen Vinschgau, Oberinntal, Graubünden, Schwaben und Bayern. Landeck, Bezirksmuseum Landeck.

Statistik Austria (Hrsg.), Volkszählung 2001. - http://www.statistik.at

Statistik Schweiz (Hrsg.), Volkszählung 2000. - http://www.bfs.admin.ch

Steinicke E. (1991), Friaul - Friuli. Bevölkerung und Ethnizität (= Innsbrucker Geogr. Studien, 19). Innsbruck, Inst. f. Geogr. d. Univ. Innsbruck.

Steinicke E. (2001a), Städte als Innovationszentren für eine Neubesinnung ethnischer Minderheiten. Das Beispiel der Kärntner Slowenen (Österreich). In: Geographica Helvetica, 56, S. 249-260.

Steinicke E. (2001b), Die Kärntner Slowenen und die Südtiroler Ladiner. Eine Renaissance kleiner ethnischer Minderheiten? In: Berichte z. dt. Landeskunde, 75, S. 395-415.

Steinicke E. (2002), Erhalt und Verfall ethnischer Minderheiten. Das Beispiel der Slowenen in Kärnten und Friaul. In: KraAs F., Stadelbauer J. (Hrsg.), Nationalitäten und Minderheiten in Mittel- und Osteuropa (= Ethnos, 60), S. 118-132. Wien.

Steinicke E. (2005), Von der „ethnischen Gruppe“ zur „ethnischen Minderheit“. Ein Beitrag zur Ethnoterminologie. In: GW-Unterr., 98, S. 31-38.

Steinicke E., ZupančIČ J. (1994), Die Kärntner Slowenen. Eine Volksgruppe der Peripherie? In: GW-Unterr., 53, S. 40-56.

Steinicke E., ZupanČIČ J. (1995), Koroški Slovenci v luči sodobnih prostorskih, socialnih in etničnih procesov [Die Kärntner Slowenen im Lichte moderner räumlicher, sozialer und ethnischer Prozesse]. In: Razprave in gradivo/Treaties and Documents, 29-30, S. 111-126.

Steinicke E., Piok E. (2003), Die deutschen Sprachinseln im Süden der Alpen. Problematik und Konsequenzen der besonderen ethnischen Identifikationen am Beispiel von Gressoney und Tischelwang (Italien). In: Berichte z. dt, Landeskunde, 77, S. 301-327.

Steininger R. (2003), Südtirol. Vom Ersten Weltkrieg bis zur Gegenwart. Innsbruck, Studienverlag. StraKa M. (Hrsg.) (1970), Handbuch der europäischen Volksgruppen. Wien, Braumüller.

Telmon T. (1992), Le minoranze linguistiche in Italia. Alessandria, Edizione dell‘Orso.

Toso F. (2008), Le minoranze linguistiche in Italia. Bologna, Molino.

UNKART R. (1997), Sind die Slowenen in der Steiermark eine eigene Volksgruppe? In: ANDERWALD K., Krapf P., SMrtnik V. (Hrsg.), Sprachminderheiten: Herausforderung und Chance (= Kärnten Dokumentation, 15), S. 121-135. Klagenfurt, Land Kärnten.

Unkart R., Glantschnig G., Ogris A. (1984), Zur Lage der Slowenen in Kärnten. Die slowenische Volksgruppe und die Wahlkreiseinteilung 1979 - eine Dokumentation. Klagenfurt, Kärntner Landesarchiv.

VAVTI S. (2005), “Wir sind Kanaltaler!” - Regionale und lokale Identitäten im viersprachigen Valcanale in Italien. In: Forum Qualitative Sozialforschung (Online Journal), 7(1), Art.34. - http://www.qualitative-research.net/index.php/fqs/article/view/78 (Mai 2011).

V AVTI S. (2007), Asimilacija in identitete v Kanalski dolini in na Južnem Koroškem - primerjalni pregled [Assimilation und Identität im Kanaltal und in Südkärnten - Übersicht anhand von Beispielen]. In: Razprave in gradivo/Treatises and documents, 53-54, S. 166-177.

Veiter T. ( $\left.{ }^{2} 1984\right)$, Nationalitätenkonflikt und Volksgruppenrecht im ausgehenden 20. Jahrhundert, Bd. 1. München, Braumüller.

W ALDER J., LÖFFler R., STEINICKE E. (2010), Autochthone ethno-linguistische Minderheiten in den italienischen Alpen im Lichte des aktuellen demographischen Wandels. In: Europa Regional, 16 (2008), S. 178-190.

ZÜRRER P. (1999), Sprachinseldialekte. Walserdeutsch im Aostatal (Italien) (= Sprachlandschaft, 23). Aarau, Sauerländer. 